\title{
Viscoelastic Finite-difference Modeling with Application to Shallow Seismic Refraction Data
}

\author{
Koichi Hayashi \\ OYO Corporation, Tsukuba Technical Research and Development Center \\ Tsukuba, Ibaraki, Japan
}

\begin{abstract}
I have developed a two-dimensional, viscoelastic, finite-difference modeling method for complex surface topography and subsurface geological structure. Realistic modeling of seismic wave propagation in the near surface is complicated by many factors, such as strong heterogeneity, topographic relief and large attenuation. In order to account for these complications, I use a velocity-stress staggered grid and employ an $\mathrm{O}(2,4)$ accurate viscoelastic finite-difference scheme. The implementation includes an irregular free surface condition for topographic relief. The algorithm is applied to 2-D modeling of the viscoelastic response of near surface structure beneath a 2-D refraction survey line. The P-velocity models were constructed by 2-D traveltime tomography, and S-velocity, density and Q were given empirically. Comparison of the observed waveform data with viscoelastic response clearly demonstrates the importance of inclusion of viscoelasticity. The character of the observed waveform data can be explained by velocities and $Q$ distributions.
\end{abstract}

\section{2-D Viscoelastic Finite-Difference Method}

We have developed 2-D viscoelastic finite-difference method that employs an $O(2,4)$ accurate viscoelastic staggered finite-difference scheme (Hayashi, 1999b; Hayashi and Bums, 1999a,b). The implementation includes an irregular free surface condition for topographic relief. In the free surface condition, stresses are calculated so that the shear and normal stresses perpendicular to the boundary are zero. The calculation of particle velocities does not involve any specific boundary conditions, and the particle velocities are set to zero above the free surface. Numerical tests indicate that approximately 30 grid-points per shortest wavelength results in accurate calculations. The method is accurate and stable, and allows us to handle complex structure in finite-difference modeling.

\section{2-D Seismic Refraction Data}

A two-dimensional seismic refraction method was performed in the mountainous area of Japan. The dynamite, placed at the depth of about $1 \mathrm{~m}$, was used as energy source. The quantity of dynamite was approximately 100 to $200 \mathrm{~g}$. The waveform data were collected at the receivers placed on the surface. The velocity seismometers (OYO SMC70 geophone) that have the natural frequency of $28 \mathrm{~Hz}$ were used as the receivers, and an OYO DAS- 1 was used as the data acquisition system. The receivers record the vertical component of particle velocities.

First arrival traveltimes were picked and the non-linear traveltime tomography (Hayashi and Saito, 1996; Hayashi, 1999a) was applied to the traveltime data. The results of the tomography (P-wave velocity structures) are shown in Figure 1. The traveltime tomography of refraction data has non-uniqueness, and therefore, I can show several velocity models those satisfy the observed traveltime data. Figure 1 (a) shows a velocity model with the assumption that a velocity structure is a three-layer model. Figure 1 (b) shows a velocity 
model with the assumption that a velocity increases smoothly with depth. Figure 2 shows the comparison of the observed and theoretical traveltime curves. One can see that both velocity models satisfy the observed data with a smaller error than that due to traveltime picking. One can not decide which velocity model is true, as long as we use only the first arrival travel times.

Hayashi (1999) showed the large difference of waveforms between layered and smooth models. In the following section, I will calculate the theoretical waveforms for both layered and smooth velocity models, and compare the theoretical and observed waveforms.

\section{Model Building for Viscoelastic Finite-difference Calculation}

Since I have an only P-velocity model, I have to assume other material properties. Svelocity is assumed to be the $1 / \sqrt{3}$ of P-velocity (Poisson solid). A density is assumed to be constant $2000 \mathrm{~kg} / \mathrm{m}^{3}$. P-wave sources are placed at the depth of $1 \mathrm{~m}$, and a $50 \mathrm{~Hz}$ Ricker wavelet is used as a source wavelet. The center frequency of the source wavelet is chosen from the frequency of the observed data. The simulations are performed for two cases. The first simulation uses a model in which the $Q_{p}$ and $Q_{s}$ are set to constant 10000 . The second simulation uses a model in which the $\mathrm{Q}_{\mathrm{p}}$ and $\mathrm{Q}_{\mathrm{s}}$ are set to 5 in the region where the $\mathrm{P}$-velocity is lower than $2000 \mathrm{~m} / \mathrm{sec}$, and 100 in the region where the P-velocity is higher than $2000 \mathrm{~m} / \mathrm{sec}$. Since the $Q$ is generally very small in the near surface region of a mountainous area, the later model is more realistic than the first one.

In the smooth model, velocity model is represented by 30 by 15 (horizontal by vertical) arbitrary quadrilateral cells. In the finite-difference calculations, the grid size of $0.125 \mathrm{~m}$ is used and the model $(150 \mathrm{~m}$ by $100 \mathrm{~m})$ is gridded into 1200 by 800 grids. Since the absolute amplitude of waveforms highly depends on the coupling between geophones and the ground, I will not be concerned with absolute amplitude of waveforms in this comparison, but will be concerned only with the general appearance.

\section{Comparison of Observed and Theoretical Waveform Data}

Figure 3 shows the observed common source data with the source at the distance of 122.5m. In Figure 3, waveforms are normalized by each trace. One can clearly see first arrivals (P-waves) and the later low frequency arrivals. The continuity of the later arrivals implies that the model has no sharp layer boundary. Figure 4 (a) and (b) show theoretical waveforms for the layered model. Regardless of the attenuation, the appearance of waveforms differs from the observed data. The theoretical waveforms are much scattered in comparison to the observed data. Figure 4 (c) and (d) show the theoretical waveforms for the smooth model. The waveforms for a small $Q$ model (d) are more similar to the observed data in comparison to those of the layered model. This result strongly suggests that the true model is a smooth one. One can see several obvious characters in the observed data, such as the first arrival traveltimes, the frequency difference between the first arrival and the later arrival, the amplitude ratio of the first arrival and the later arrival, the apparent velocity of the later arrival, and complicated waveforms at smaller distances ( 0 to $40 \mathrm{~m}$ ). One can see that the theoretical waveforms of the smooth model with small $Q$ have similar characters.

\section{Conclusions}

It was possible to obtain synthetic seismograms that were close to observed field data. I can say that the model used in the simulation was not far from the true structure. This 
simulation should be considered as a preliminary step. In order to perform complete modeling, we need to avoid the assumptions, build a three-dimensional model, and calculate threedimensional wave propagation. Such a complete modeling is possible, but extremely expensive and time consuming at this time. However, the comparison of the waveforms for the layered model with the smooth model showed that the finite-difference modeling could be a strong analysis tool for shallow seismic methods, in spite of the assumptions.

\section{References}

Hayashi, K. and Saito, H., 1996, Automatic correction of velocity structures in seismic refraction prospecting, 66th Annual International Meeting, Soc. Expl. Geophys., Expanded Abstracts, 1658-1661.

Hayashi, K., 1999a, Application of high resolution seismic refraction method to civil engineering investigations, 61st EAGE Conference \& Technical Exhibition, Extended Abstracts, 4-46.

Hayashi, K., 1999b, Variable grid finite-difference modeling including surface topography, M.Sc. Thesis, Massachusetts Institute of Technology.

Hayashi, K., and Burns, D. R., 1999a, Variable grid finite-difference modeling including surface topography, Borehole Acoustic and Logging and Reservoir Delineation Consortia, Annual Report 1999, Earth Resources Laboratory / MIT.

Hayashi, K., and Bums, D. R., 1999b, Variable grid finite-difference modeling including surface topography, 69th Annual International Meeting, Soc. Expl. Geophys. Expanded Abstracts, 528-531.

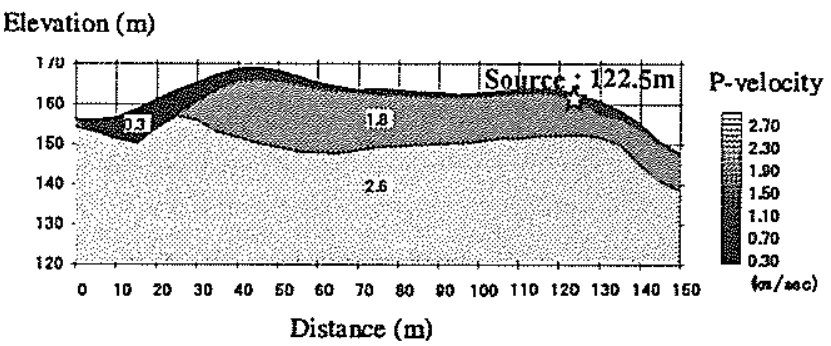

a) Three-layer model

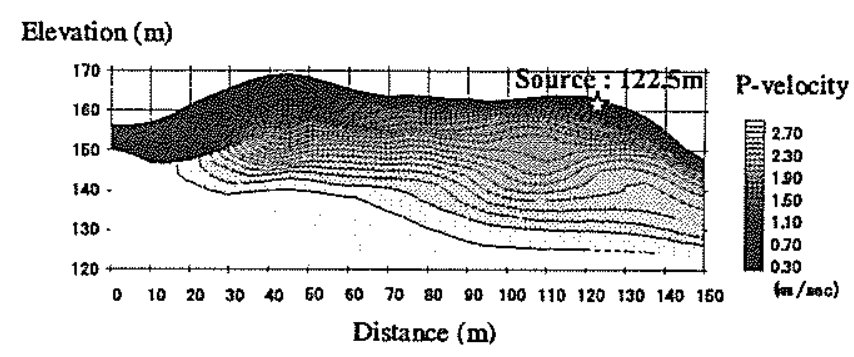

b) Smooth model

Figure 1. P-velocity tomogram obtained by the refraction traveltime tomography. a) A velocity model with the assumption that the velocity structure is a three-layer model. b) A velocity model with the assumption that the velocity is increasing with depth smoothly.

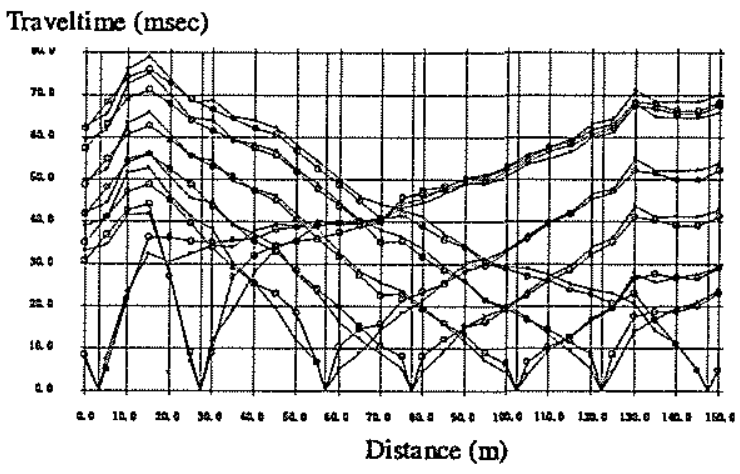

a) Three-layer model (Figure 1a)

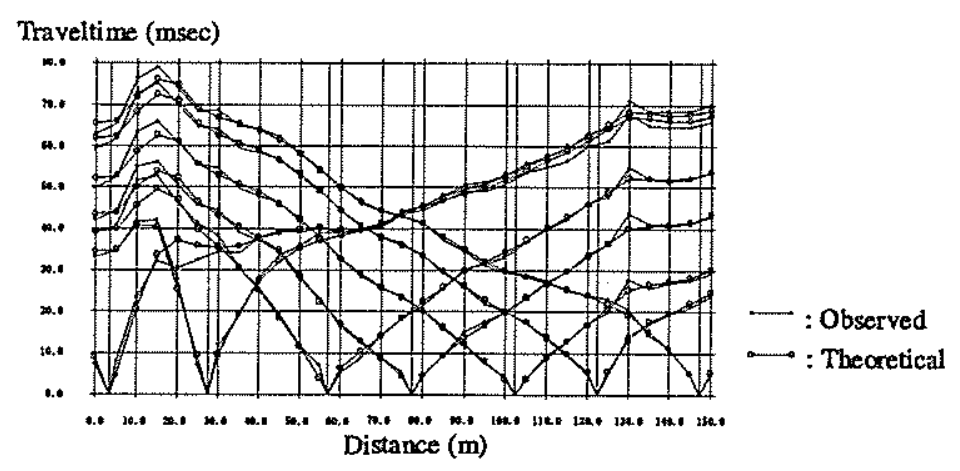

b) Smooth model (Figure 1b)

Figure 2. Comparison of the observed and theoretical traveltime data. 


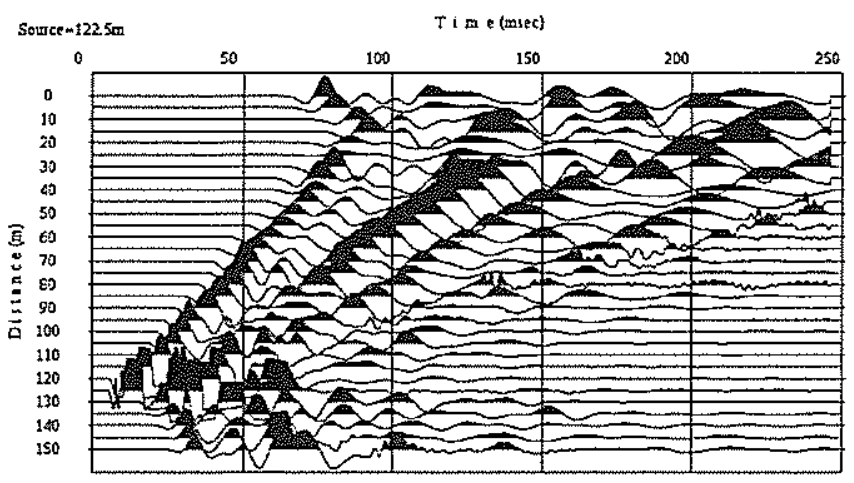

Figure 3. Example of observed common source data. A source location is $122.5 \mathrm{~m}$. A source depth is about $1 \mathrm{~m}$. The quantity of dynamite was $100 \mathrm{~g}$. A particle velocity (vertical component) is plotted.

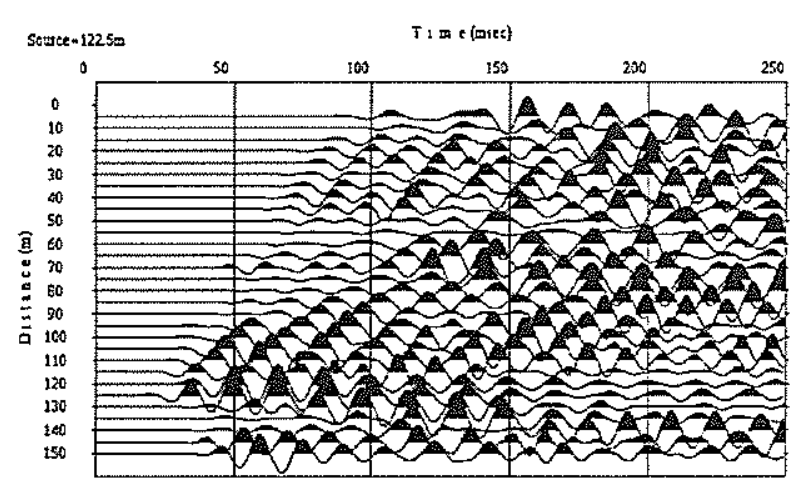

a) Three layer model. $Q=10000$.

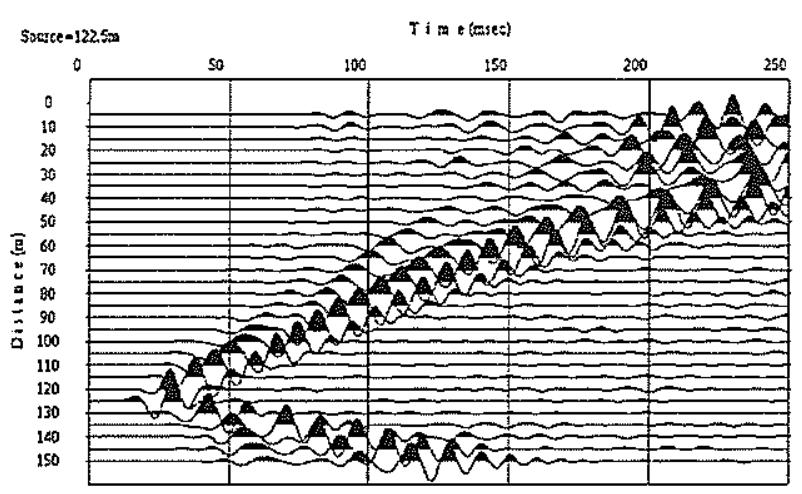

c) Smooth model. $Q=10000$.

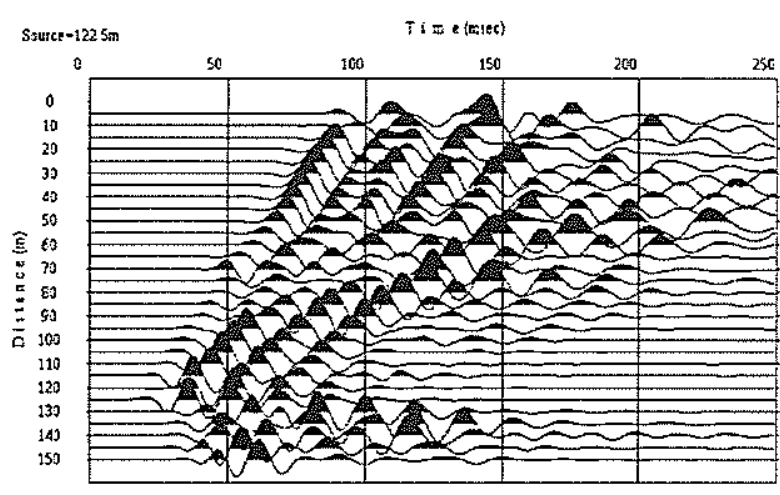

b) Three layer model. $Q=5(\mathrm{Vp}<2000 \mathrm{~m} / \mathrm{sec})$ and $\mathrm{Q}=100(\mathrm{Vp} \geqq 2000 \mathrm{~m} / \mathrm{sec})$.

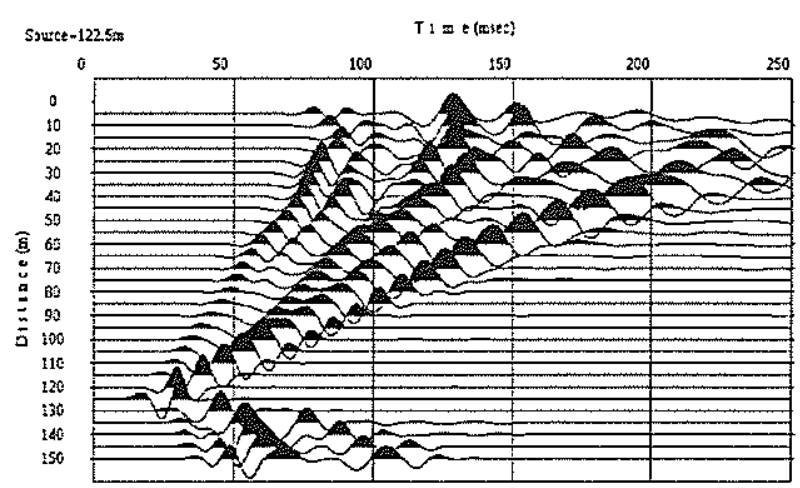

d) Smooth model. Q=5 $(\mathrm{Vp}<2000 \mathrm{~m} / \mathrm{sec})$ and $\mathrm{Q}=100(\mathrm{Vp} \geqq 2000 \mathrm{~m} / \mathrm{sec})$.

Figure 4. Theoretical data calculated by the finite-difference method. Vertical component of particle velocity is plotted. 